\title{
Irreversible Bone Marrow Failure after Cladribine Treatment in a Patient with Hairy Cell Leukemia
}

\author{
Eugenio Galli*, Marianna Criscuolo, Luana Fianchi, Andrea P. Bacigalupo and Livio Pagano \\ Istituto di Ematologia, Polo di Onco-Ematologia, Fondazione Policlinico Agostino Gemelli Università Cattolica del Sacro Cuore
}

Received: April 26, 2018; Published: May 08, 2018

*Corresponding author: Dott Eugenio Galli, Istituto di Ematologia, Polo Di Onco-EmatologiaFondazione Policlinico A Gemelli-Università Cattolica del Sacro Cuore, Largo F. Vito 1 I-0068 Roma Italia

\begin{abstract}
Here we describe a 67 year old female affected by hairy cell leukemia (HCL) treated with 2-CDA, who developed prolonged bone marrow failure. Severe and persistent pancytopenia led to lethal pulmonary aspergillosis on day +85 of treatment. We have then reviewed literature in order to better understand occurrence of neutropenia and infections in this kind of patients. The most prominent side effect of 2CDA has been reported to be myelosuppression, but only one irreversible failure has been described until now.
\end{abstract}

Keywords: Cladribine; Hairy Cell Leukemia; Aspergillosis; Aplasia

\section{Introduction}

Hairy cell leukemia (HCL) is B-cell lymphoproliferative disease representing $2 \%$ of all leukemias. It is considered an indolent cancer, mostly diagnosed in male patients over the age of 60 years, presenting with unexplained cytopenias. The main clinical manifestations depend on the degree of bone marrow infiltration and splenomegaly, which can occur in up to $90 \%$ of cases. The pathologic clone is a lymphoid B-cell with typical surface projections, producing a "hairy cell" morphology . Recently BRAF mutation has been described in the majority of patients. Cladribine (2-CDA), a purine analogue is considered an effective therapeutic approach, with up to $90 \%$ complete remissions after only one course of treatment.

\section{Case Report}

A 67-year-old female was seen in another Center in September 2016, because of a mild thrombocytopenia, neutrophil/lymphocyte inverted ratio, without absolute lymphocytosis, anemia or neutropenia. Morphologic, cytofluorimetric and molecular biology tests on bone marrow samples were diagnostic for BRAF-V600E mutated, kappa-clonal, CD305 and CD123 positive classical HCL. There was no splenomegaly. Because of mild alterations of peripheral blood counts and in the absence of major infections, the patient was initially placed in follow up.Three months later the woman was hospitalized for 30 days for a severe pneumonia, where she received non-invasive ventilation and was successfully treated with intravenous antibiotics and steroids.
One month after that episode (December 2016), the patient was admitted to our hematology Unitbecause of fever with chills. Her blood count was as follows: hemoglobin ( $\mathrm{Hb}) 10.1 \mathrm{~g} / \mathrm{dL}$, total white blood cells count (WBC) 700/mcL, absolute neutrophil count (ANC) 40/mcL, monocytes 0/mcL and platelets (PLTs) 113000/mcL. Biochemical tests including procalcitonine and LDH were normal. No fever was observed since the admission. Antero-posterior chest X-rays revealed a small rounded opacity. A bone marrow biopsy was repeated and showed a reduced cellularity, with $70 \%$ of atypical "hairy" lymphoid elements and increased reticular fibrosis. Immunophenotyping was consistent with the previous assessment of CD103/ CD25/CD11c/CD197/ CD25/ CD45/ CD103/Kappa chains positive classical HCL. A lung CT-scan confirmed a small resolving pulmonary consolidation. No splenomegaly was observed.

After oneweek of intravenous broad spectrum antibiotic therapy and defervescence, treatment with i.v. Cladribine $0.09 \mathrm{mg} / \mathrm{kg} / \mathrm{die}$ for 7 days was started, [1-5] on the basis of the infectious complications and severe neutropenia. Prophylaxis with posoconazole and trimethoprim/sulfamethoxazole was also administered. On day +7 of 2CDA therapy, a febrile episode was treated empirically with piperacillin/tazobactam and amikacin. On day +9 , G-CSF was added because of a total white blood cell count of $0.02 \times 10^{\wedge} 9 / \mathrm{L}$. A chest CT-scan performed on day +25 , showed multiple centrolobular confluent flogistic nodules. Thrombocytopenia (<20000/mcL) developed on day +25 from 2CDa treatment, and the patient was dependent on red cells and platelet transfusions thereafter Several 
combinations of antibiotical and antimicotic intravenous therapy, were unsuccessfully administered, with persistent negative microbiological tests. The peripheral blood counts continued to be low: on day +39 they were WBC $10 / \mathrm{mcL}$, Hb $8.9 \mathrm{~g} / \mathrm{dl}$, PLTs $9000 / \mathrm{mcL}$. A bone marrow biopsy was repeated, revealing severe fibrosis with poor cellularity composed by leukemic elements and mastcells, together with severe hypoplasia.

On day +37 Beta-D-glucane became positive in serum $(370$ pg/ $\mathrm{mL}$ ), and a broncho-alveolar lavage was performed on day +39 , which proved galactomannan positive $(\mathrm{GM})(>2.0 \mathrm{pg} / \mathrm{mL})$, and also GM in serum, previously always negative, became positive $(>2.3$ $\mathrm{pg} / \mathrm{mL}$ ), despite empirical liposomal amphotericin B (L-AmB) from day +22 . The patient remained febrile with occasional exacerbation and chills, while neutrophils count, hemoglobin and platelet count remained steadily mirroring the underlying aplasia (Figure 1). On day +49 a further marrow biopsy was performed, and showed moderate fibrosis, poor cellularity, with $50 \%$ of B lymphocytes CD20/79a+, rare erythroid and megakaryocytic elements, and absence of granulocytopoiesis. Molecular search for BRAF V600E was positive [5-7].

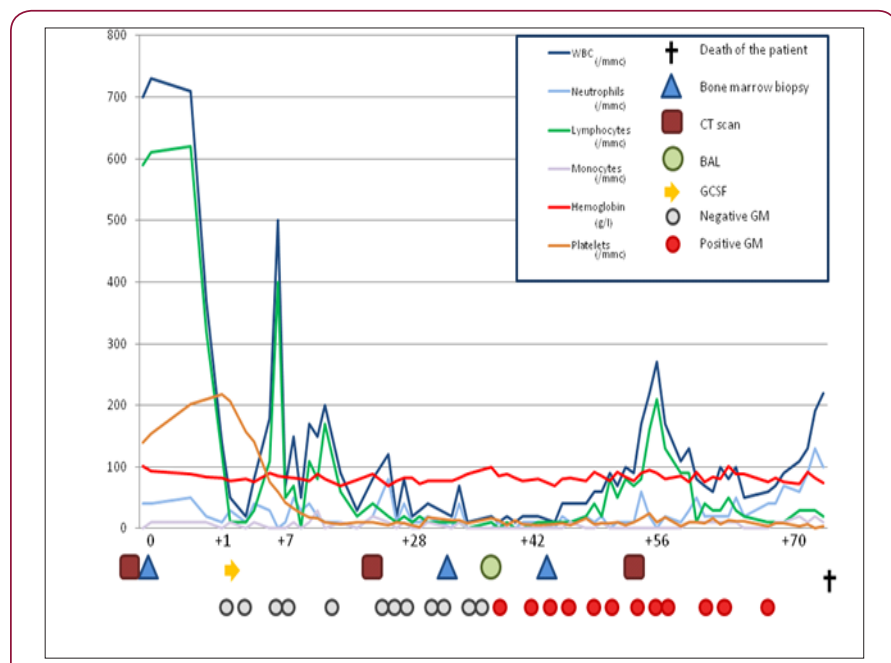

Figure 1: Main clinical, hematological and microbiological findings.

ACT-scan performed on day +58 was consistent with pulmonary aspergillosis and L-AmB was replaced by voriconazole. We later assisted to a progressive worsening of the clinical conditions, with crackles, rales, rust-colored sputum and increased oxygen need. On day +73 hepatotoxicity occurred, as the initial sign of a multi-organ failure. The clinical conditions of the patient further deteriorated on day +76 , the patient required high flow oxygen support and was transferred to intensive care unit (ICU). On day +82 the hemoglobin was $8.2 \mathrm{~g} / \mathrm{dL}$, WBC 190/mcL, ANC 130/mcL, monocytes 30/mcL, PLTs $1.000 / \mathrm{mcL}$. The patient died on day +85 of 2CDa treatment.

\section{Discussion}

Consensus Guidelines for HCL recommend delaying leukemiaspecific therapy if an active infection is underlying. If attempts to control the infection fail and leukemia-specific treatment is needed, purine analogue or interferon alpha can be used, despite reasonable higher risk of myelosuppression.Since the advent of cladribine, the course of HCL has improved dramatically, and around $80 \%$ of complete remission may be obtained with a single course of therapy.

As to infections,HCL is widely known to be associated with defective immune function, both before and after therapy. A well assessed biological landscape of humoral and cellular alterations are responsible for pre-treatment immunodeficiency, such as impaired antigen presentation, monocytopenia, NK and T-cell defective activity and high serum levels of IL2 receptor.In addition to this, purine analogue administration may contribute to infective risk with different mechanisms. Cladribine administration was reported to induce a decreased CD8+ T cells and NK cells count for 3 o 4 months after the end of treatment, while deep CD20+ and CD4+ lymphocytopenias are commonly observed for more than 2 years. Neutrophils nadir often occurs during second week of treatment, while neutropenic fever is reported in $40-50 \%$ of cases, with documented infection only in 10-15 \% of cases. The average time to $\mathrm{ANC}>1000 / \mathrm{mmc}$ is $9-22$ days.

Infectious complications during the purine-analogue treatments are rarely reported and usually happen during the aplastic phase after chemotherapy. In 2009 Else et al. described 233 patients with HCL treated with pentostatin or cladribine as single agents: by analyzing the group of cladribine-treated patients (45 cases), 1-year-OS was $100 \%$, no grade 3-4 infectious complications were reported.Another study on a group of 50 patients treated with Cladribrine reported only 1 case of fatal septic shock.On the contrary, in 2011 Ravandi et al. [8] tested a 5-day regimen of 2-CDA, followed by Rituximab in 36 patients with HCL and reported $33 \%$ of grade 3 or 4 infections, including neutropenic fever, cellulitis and herpes zoster dermatitis, but not fungal infections. . In accordance, Tadmor et al. [9] reported a cumulative incidence of infections in $30-50 \%$ of patients during the first 4-6 weeks after treatment. It is probable that the addition of Rituximab increased the infectious risk.

While prolonged lymphopenia after cladribine treatment is usually reported, cases of irreversible leucopenia with the onset of aplastic anemia are really rare. To the best of our knowledge only 1 case of bone marrow aplasia in HCL has been reported until now. In 2011 a case of bone marrow aplasia after treatment with 2-CDA following H1N1 pneumonia was reported. Anyway that case was different than the one we describe, since cytopenias at diagnosis were not severe, G-CSF was administered as prophylaxis and aplasia occurred 3 weeks after the end of treatment. In addition to this, and differently from the case we report, bone marrow biopsy after treatment was leukemia free, weather in our report the patient seemed to have residual clonal cells.

In our report, a major and a minor lung-infective event occurred before the admission in our Deparment, although fever was not present. Despite a slight nodular lung image in the chest X-ray, the absence of fever and the marked cytopenias were sufficient to decide for starting the treatment. By comparing the two cases, it is possible to hypothesize that our patient had suffered profound toxic myelosuppression, possibly over-exacerbated by an infection. Moreover, she did not achieve control of her HCL, with $50 \%$ 
marrow infiltration after 4-6 weeks from treatment, together with persistence of BRAF V600E expression. The prolonged and deep neutropenia favored the onset of a pulmonary aspergillosis more frequently observed in patients with acute leukemia. The invasive aspergillosis, in combination with persistent disease and bone marrow failure, was responsible ofthe death of our patient [10-13].

Despite of this unfortunate patient, in our opinion a full dose treatment should be administered in HCL with moderate-to-severe cytopenias or recurrent infections. When treating HCL with purine analogues, infective adverse events are frequent and may need careful management often requiring hospitalization. Clinicians should not be discouraged by episodic fatal events, though great attention needs to be given to recent infective episodes, which may synergize with profound myelosuppression induced by the disease and 2CDA treatment.

\section{References}

1. Grever MR, Abdel-Wahab O, Andritsos LA, Barrientos J, Blachly JS, et al. (2017) Consensus guidelines for the diagnosis and management of patients with classic hairy cell leukemia. Blood 129(5): 553-560.

2. Tiacci E, Trifonov V, Schiavoni G, Antony Holmes, Wolfgang Kern, et al. (2011) BRAF mutations in hairy-cell leukemia. The N E J med 364(24): 2305-2315.

3. Khorshid O, Namour AE, El-Gammal MM, Mahmoud TY, Fortpied C, et al. (2015) Efficacy and Safety of Cladribine: Subcutaneous versus Intravenous Administration in Hairy Cell Leukemia Patients. Mediterr J Hematol Infect Dis 7(1): 7.

4. Dasanu CA, Ichim TE, Alexandrescu DT (2009) Inherent and iatrogenic immune defects in hairy cell leukemia: revisited, Expert Opinion on Drug Safety 9(1): 55-64.

5. Zenhäusern R, Schmitz SF, Solenthaler M, Rufener B, Tobler A, et al. (2009) Randomized trial of daily versus weekly administration of 2-chlorodeoxyadenosine in patients with hairy cell leukemia: a multicenter phase III trial (SAKK 32/98). Leukemia \& Lymphoma 50(9): 1501-1511.

6. Saven A, Burian C, Adusumalli J,Koziol JA (1999) Filgrastim for Cladribine-Induced Neutropenic Fever in Patients With Hairy Cell Leukemia Blood 93(8).

7. Else M, Dearden CE, Matutes E, Lauria F, Catovsky D, et al. (2009) Longterm follow-up of 233 patients with hairy cell leukaemia, treated initially with pentostatin or cladribine, at a median of 16 years from diagnosis. British journal of haematology 145(6): 733-740.

8. Ravandi F, O'Brien S, Jorgensen J, Thomas D, Keating M, et al. (2011) Phase 2 study of cladribine followed by rituximab in patients with hairy cell leukemia Blood 118(14): 3818-3823.

9. T Tadmor (2011) Purine analog toxicity in patients with hairy cell leukemia. Leukemia and Lymphoma 52(Suppl. 2): 38-42.

10. Helbig G, Wozniczka K, Wieczorkiewicz A, Pajak J, Kyrcz-Krzemien $S$ (2011) Irreversible marrow aplasia after single course of 2-chlorodeoxyadenosine for hairy cell leukaemia preceding by A pandemic 2009-H1N1-associated pneumonia. Medical Oncology 28 (4): $1601-1603$

11. Caira M, Candoni A, Verga L, Rossi G, Spadea A, et al. (2015) Prechemotherapy risk factors for invasive fungal diseases: prospective analysis of 1,192 patients with newly diagnosed acute myeloid leukemia (SEIFEM 2010-a multicenter study).Haematologica 100(2): 284-292.

12. Juliusson G, Samuelsson H (2011) Hairy cell leukemia: epidemiology, pharmacokinetics of cladribine, and long-term follow-up of subcutaneous therapy. Leukemia \& Lymphoma 52(Suppl. 2): 46-49.

13. Leupin N, Schuller JC, Solenthaler M, Hanselmann S, Zenhäusern R.et al. (2010) Efficacy of rituximab and cladribine in patients with chronic lymphocytic leukemia and feasibility of stem cell mobilization: a prospective multicenter phase II trial (protocol SAKK 34/02).]. Leukemia \& Lymphoma 51(4): 613-619.

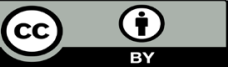

This work is licensed under Creative Commons Attribution 4.0 License

Submission Link: https://biomedres.us/submit-manuscript.php

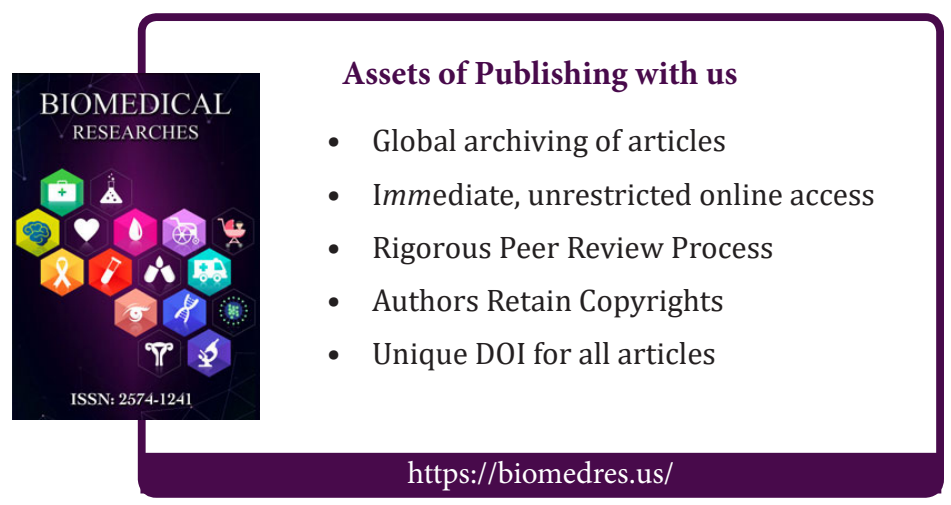

